\title{
Aberrant expression of FoxP3 in a human T cell line possessing regulatory $T$ cell-like function and exposed continuously to asbestos fibers
}

\author{
MEGUMI MAEDA ${ }^{1,2}$, HIDENORI MATSUZAKI ${ }^{2,3}$, SHOKO YAMAMOTO ${ }^{2}$, SUNI LEE ${ }^{2}$, \\ NAOKO KUMAGAI-TAKEI ${ }^{2}$, KEI YOSHITOME ${ }^{2}$, YU MIN ${ }^{2,4,5}$, NAGISA SADA ${ }^{2,6}$, \\ YASUMITSU NISHIMURA $^{2}$ and TAKEMI OTSUKI ${ }^{2}$
}

\author{
${ }^{1}$ Department of Biofunctional Chemistry, Division of Bioscience, \\ Okayama University Graduate School of Natural Science and Technology, Kita-Ku, Okayama 700-8530; \\ ${ }^{2}$ Department of Hygiene, Kawasaki Medical School, Kurashiki, Okayama 701-0192; \\ ${ }^{3}$ Department of Life Science, Faculty of Life and Environmental Science, Prefectural University of Hiroshima, \\ Shobara, Hiroshima 727-0023, Japan; ${ }^{4}$ Department of Occupational and Environmental Health Sciences, \\ School of Public Health, Peking University, Beijing 100191; ${ }^{5}$ Department of Occupational Diseases, \\ Zhejiang Academy of Medical Sciences, Hangzhou, Zhejiang 310013, P.R. China; \\ ${ }^{6}$ Department of Biophysical Chemistry, Graduate School of Medicine, \\ Dentistry and Pharmaceutical Sciences, Okayama University, \\ Okayama 700-8530, Japan
}

Received January 13, 2018; Accepted June 4, 2018

DOI: $10.3892 /$ or.2018.6481

\begin{abstract}
Prompted by the known carcinogenic activity of asbestos, our investigations revealed that asbestos causes a reduction in antitumor immunity. One mechanism involves the enhancement of regulatory $\mathrm{T}$ (Treg) cell function and volume assayed using MT-2 original cells (Org), an HTLV-1 immortalized human $\mathrm{T}$ cell line which possesses Treg-like function. Continuous and relatively low-dose exposure of MT-2 to asbestos fibers yielded sublines resistant to asbestos-induced apoptosis and enhanced Treg function via cell-cell contact mechanisms and increased the production of soluble factors such as interleukin (IL)-10 and transforming growth factor (TGF)- $\beta$. Additionally, cell cycle progression was accelerated in these sublines. Subsequently, the status of the Treg-specific transcription factor FoxP3 was examined. Unexpectedly, FoxP3 mRNA levels decreased in the sublines, although significant changes in protein expression were absent. Methylation analysis of $\mathrm{CpG}$ sites located in the promoter region of FoxP3 in original MT-2 cells and sublines showed almost complete methylation in Org and slight hypomethylation in the sublines. Although treatment with the demethylating agent
\end{abstract}

Correspondence to: Professor Takemi Otsuki, Department of Hygiene, Kawasaki Medical School, 577 Matsushima, Kurashiki, Okayama 701-0192, Japan

E-mail: takemi@med.kawasaki-m.ac.jp

Key words: asbestos, regulatory $\mathrm{T}$ cell, FoxP3, methylation 5-aza-deoxycytidine tended to upregulate FoxP3 expression, the methylation status did not match the mRNA expression and enhanced function. Additionally, the expression of other transcription factors related to Treg did not differ between Org and subline CB1. Collectively, aberrant expression and methylation patterns of FoxP3 were detected in human T cells continuously exposed to asbestos, although cell function was enhanced by asbestos exposure. Future analyses to identify factors responsible for Treg functional enhancements induced by asbestos, such as the investigation of surface molecules, are needed for the development of strategies to prevent the occurrence of asbestos-induced cancers.

\section{Introduction}

Asbestos fibers promote the incidence of malignant tumors such as lung cancer and malignant mesothelioma (MM) (1-3). Additionally, the International Agency for Research on Cancer (IARC) has categorized asbestos as causing laryngeal and ovarian cancers (4). The carcinogenic mechanisms at play resulting from exposure to asbestos fibers are thought to mainly involve DNA damage via a Fenton reaction which produces reactive oxygen species (ROS) induced by the iron included in fibers such as crocidolite and amosite (5-7). Additionally, ROS are also produced from alveolar macrophages (AMs), the latter being involved in the initial response to foreign substances such as the recognition and digestion of foreign substances. However, AMs are unable to completely phagocytize asbestos fibers due to fiber rigidity and size. Thus, AMs become frustrated and produce ROS $(8,9)$. These ROS cause DNA damage in surrounding lung epithelial cells as well as facilitate the 
transformation of pleural mesothelial cells toward malignant transformation. Furthermore, since cells possess the tendency to incorporate fibers, the fibers can damage chromosomes directly due to fiber rigidity and shape $(10,11)$. Additionally, inhaled asbestos fibers remaining in the pulmonary fields persistently adsorb various carcinogenic chemicals included in tobacco smoke and air pollution $(10,11)$. All of these factors are considered to play a role in the carcinogenic potential of asbestos fibers.

We have been investigating the immunological effects of asbestos fibers (12-14). The activity of natural killer (NK) cells was found to be reduced in asbestos-exposed patients with pleural plaque (PP) or MM $(15,16)$. In particular, the cell killing activity of receptor NKp46 was found to be reduced in NK cells derived from patients with PP or MM $(15,16)$. Additionally, clonal proliferation and differentiation of $\mathrm{CD}^{+}$ cytotoxic T lymphocytes (CTLs) monitored by the mixed lymphocyte reaction (MLR) were reduced when chrysotile fiber was added to the MLR cultures (17). It was then found that CXCR3, a chemokine receptor important for antitumor immunity, was reduced in cells continuously exposed to asbestos such as the human T cell leukemia virus (HTLV)-1 immortalized human polyclonal T cell line, MT-2, freshly isolated $\mathrm{CD}^{+} \mathrm{T}$ cells from healthy volunteers activated in vitro with asbestos fibers, as well as peripheral $\mathrm{CD}^{+} \mathrm{T}$ cells derived from PP and MM patients $(18,19)$. All of these findings indicate that continuous exposure to asbestos causes antitumor immunity and may set the background for the occurrence of cancer in asbestos-exposed individuals (12-14).

From the viewpoint of antitumor immunity, the function and volume of regulatory T (Treg) cells is also important (20-24). Since the MT-2 cell line possesses Treg function $(23,24)$, we observed the effects of asbestos exposure on MT-2 cells. Initially, transient and relatively high-dose exposure, which induced growth suppression, caused apoptosis via ROS production and activation of the mitochondrial apoptotic pathway (Fig. 1) (25). Then, continuous and relatively low-dose exposure, which affected less than half of the cells affected by transient exposure, for more than eight months using chrysotile B (CB), chrysotile A (CA) and crocidolite (CR) fibers resulted in the alteration of certain cellular characteristics $(26,27)$. As displayed in Fig. 1, independently established sublines were exposed to CB (designated as CB1 to 3), CA (CA1 to 3) and CR (CR1 to 4) $(18,26,27)$. All of these sublines acquired resistance to asbestos-induced apoptosis, changes in cytokine production [increase in interleukin (IL)-10 and transforming growth factor (TGF)- $\beta$ and reduction in interferon (IFN)- $\gamma$ )], and alteration of cytoskeletal molecules $(18,26-28)$. Thereafter, Treg function of the original MT-2 cells and the aforementioned sublines were examined (29). It was found that suppressive function was enhanced in sublines by cell-cell contact and an increase in soluble factors such as IL-10 and TGF- $\beta$ (29) was observed. Additionally, expression of the transcription factor forkhead box protein $\mathrm{O} 1$ (FoxO1) was lower in all sublines compared with the original MT-2 cells (30). FoxO1 acts by regulating cell cycle-related genes, with negative regulation for accelerating cyclins and positive regulation for breaking cyclin-dependent kinase-inhibitors (CDK-Is). Thus, all sublines showed increased expression of cyclins and decreased expression of CDK-Is which resulted in the acceleration of cell cycle progression (31). These findings indicate that asbestos exposure induces enhanced Treg function as well as proliferation.

In the present study, the status of transcription factor forkhead box P3 (FoxP3), regarded as one of the most important factors for Treg (20-22), as well as other Treg-specific molecules were assayed and compared with original MT-2 cells and sublines continuously exposed to asbestos in terms of expression and epigenetic conditions.

\section{Materials and methods}

Cell lines. Details of the MT-2 original (Org) cell line and the asbestos-induced apoptosis-resistant sublines have previously been reported $(26,27,32)$. These cells were maintained in a humidified atmosphere of $5 \% \mathrm{CO}_{2}$ at $37^{\circ} \mathrm{C}$ in RPMI-1640 medium (Thermo Fisher Scientific, Inc., Waltham, MA USA) supplemented with $10 \%$ fetal calf serum (FCS; Thermo Fisher Scientific, Inc.), streptomycin and penicillin (Fujifilm Wako Pure Chemical Corp., Osaka, Japan). Seven asbestos-resistant sublines, CA1-3, CB1-3 and CR1-4, were generated by continuous exposure to $\mathrm{CA}, \mathrm{CB}$, and $\mathrm{CR}$, respectively. As previously reported $(26,27,32)$, the dose of asbestos fibers utilized for continuous exposure was between 5 and $10 \mu \mathrm{g} / \mathrm{ml}$. This dose induced less than half of the cells to proceed toward apoptosis compared with cells subjected to transient exposure $(26,27,32)$. The International Union Against Cancer standards CA and ChB were kindly provided by the Department of Occupational Health at the National Institute for Occupational Health, South Africa (25-28). Additionally, CA, CB and CR were kindly provided as standard fibers from the Japan Association for the Study of Fiber Materials. Mineralogical features of the fibers used have previously been reported (33).

Real-time RT-PCR, western blotting and flow cytometry. Expression levels of Treg-related genes (20-22), FoxP3, cytotoxic T-lymphocyte-associated protein 4 (CTLA4) (34), glucocorticoid-induced TNFR-related protein (GITR) (35), IKAROS family zinc finger 2 (IKZF2) (36), special AT-rich sequence-binding protein-1 (SATB1) (37), acute myeloid leukemia 1 protein (AML1) also known as Runt-related transcription factor 1 (Runx1) or core-binding factor subunit $\alpha-2$ and DNA methylation-related genes (CBFA2) (38), and DNA (cytosine-5)-methyltransferase (DNMT)1, DNMT2, DNMT3a and DNMT3b were analyzed in Org and continuously exposed sublines (CA1-3, CB1-3 and CR1) using real-time RT-PCR assays. Total cellular mRNA from Org, CA1-3, CB1-3 and CR1 cells and 5-aza-deoxycytidine (5-aza-dC)-treated Org and CB1 cells was extracted using an RNeasy Mini kit (Qiagen GmbH, Hilden, Germany). Following synthesis of the first strand cDNA, real-time RT-PCR was performed using the SYBR-Green method (Takara Bio Inc., Otsu, Japan) with the Mx3000P qPCR System (Agilent Technologies, Inc., Santa Clara, CA, USA) according to the manufacturer's instructions as previously reported (39). Relative gene expression was calculated by the $\Delta \Delta \mathrm{Ct}$ method using an endogenous control (GAPDH) as 1.0. The formula is expressed as follows: $2^{-\Delta \Delta \mathrm{C}}=2^{-(\Delta \mathrm{Ct} \text { for target gene }-\Delta \mathrm{Ct} \text { for GAPDH })}$. All primer sequences are shown in Table I.

For western blotting, cells were lysed in $50 \mathrm{mM}$ Tris- $\mathrm{HCl}$ (pH 7.2) buffer containing $150 \mathrm{mM} \mathrm{NaCl}, 1 \%$ Nonidet P-40, 


\section{MT-2 original cell}

Treg resembling function

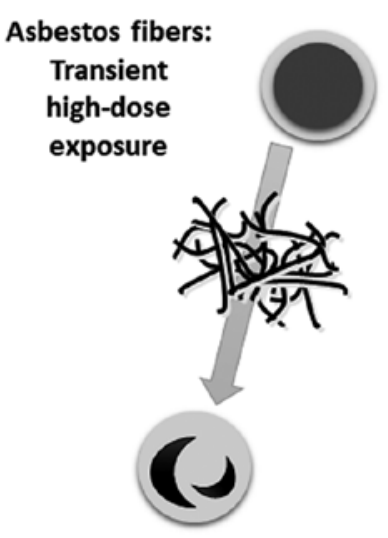

Apoptosis

- ROS production

- Activation of

mitochondrial apoptotic

pathway
Asbestos

fiber: Sublines:

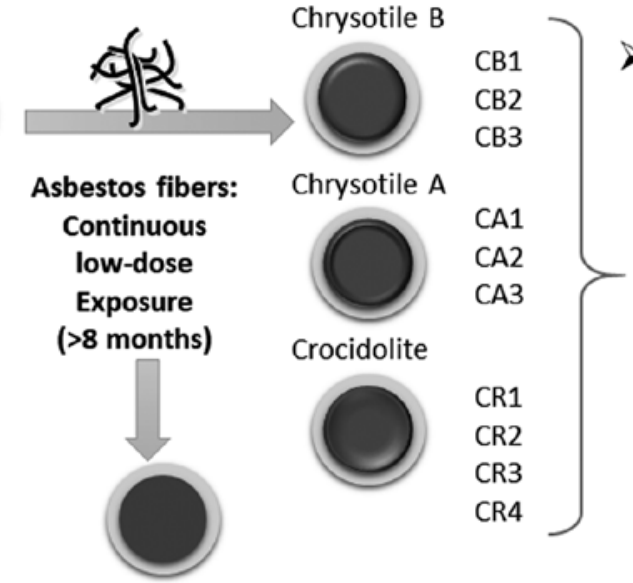

Acquisition of resistance to asbestos-induced apoptosis
Enhancement of Treg-resembling function

$\checkmark$ Increased cellcell contact suppressive activity

$\checkmark$ Increase in soluble factor production (IL10 and TGF- $\beta$ )

$\checkmark$ Acceleration of cell cycle progression

Figure 1. Schematic background of this study. A human HTLV-1 immortalized polyclonal T cell line, MT-2, was exposed to asbestos fibers (chrysotile A was initially employed, and crocidolite was then also used in other experiments) transiently and at relatively high doses. Cells proceeded to undergo apoptosis with production of ROS and activation of the mitochondrial apoptotic pathway. MT-2 cells (Org) were cultured with continuous and relatively low-dose exposure to chrysotile A, B or crocidolite for more than eight months, which induced less than half of the cells toward apoptosis. Following long-term culture, all sublines acquired resistance to asbestos-induced apoptosis. Thereafter, Treg cell function of Org and sublines was examined, since MT-2 cells are known to possess Treg-like function. As a result, all sublines showed enhanced Treg-like function via cell-cell contact as well as overproduction of soluble factors such as IL-10 and TGF- $\beta$, known to be typical factors for Treg function. Additionally, sublines showed accelerated cell cycle progression. Taken together, continuous exposure of T cells to asbestos caused enhanced Treg function and volume and these were considered as one set of factors responsible for the reduction in antitumor immunity in asbestos-exposed individuals. Treg cell, regulatory T cell; ROS, reactive oxygen species; CB, chrysotile B; CA, chrysotile A; CR, crocidolite.

$1 \%$ deoxycholic acid, $0.05 \%$ sodium dodecyl sulfate (SDS), $1 \mathrm{X}$ Protease Inhibitor Cocktail (Sigma-Aldrich; Merck KGaA, Darmstadt, Germany), and 1X Halt Phosphatase Inhibitor Cocktail (Thermo Fisher Scientific, Inc., Rockford, IL, USA). Proteins were quantified using a BCA assay kit (Thermo Fisher Scientific, Inc.), and $10 \mu \mathrm{g}$ of protein was resolved using 10\% SDS-PAGE under reducing conditions with 5\% 2-mercaptoethanol and then transferred to a PVDF membrane. Proteins were probed with the following antibodies: FoxP3 (cat. no. 14-5779-80; eBioscience; Thermo Fisher Scientific, Inc.), (cat. no. ABE-75; Millipore Sigma, Billerica, MA, USA), (cat. no. SE-21072; Santa Cruz Biotechnology, Santa Cruz, CA, USA) and actin (cat. no. 4967; Cell Signaling Technology, Danvers, MA, USA) or Histone H4 (cat. no. SC-8657; Santa Cruz Biotechnology) as control (all these antibodies were used in 1:1,000 dilution), and incubated with HRP-conjugated anti-mouse IgG or anti-rabbit IgG (cat. nos. sc-358914 and sc-2357, respectively; Santa Cruz Biotechnology) (the secondary antibody was used in 1:10,000 dilution). Proteins were detected using ECL Plus western blotting detection reagents (GE Healthcare UK Ltd., Buckinghamshire, UK).

Intracellular FoxP3 was stained using the Human Foxp3 Buffer Set and anti-FoxP3-PE antibody (clone 259D/C7; cat. no. 560046; BD Biosciences, San Diego, CA, USA) according to the manufacturer's instructions and the final concentration was $10 \mu \mathrm{g} / \mathrm{ml}$. Cells were analyzed using a FACSAria cell sorter (BD Biosciences).
Methylation analysis. Genomic DNA was isolated from Org and CB1 cells using a Qiagen DNA isolation kit (Qiagen) according to the manufacturer's instructions. Genomic DNA was digested with restriction endonuclease EcoRI or BamHI (Takara Bio Inc., Otsu, Shiga, Japan), and then used for chemical modification comprising denaturation of digested DNA with $0.3 \mathrm{M}$ sodium hydroxide for $15 \mathrm{~min}$ at $37^{\circ} \mathrm{C}$, and 3.0 $\mathrm{M}$ sodium bisulfate and $0.5 \mathrm{mM}$ hydroquinone overnight at $55^{\circ} \mathrm{C}$. Treated DNA was purified and amplified by PCR using primers listed in Table I. Amplified DNA fragments including the genomic sequence shown in Fig. 2 was subjected to cloning using the TOPO-TA Cloning kit (Thermo Fisher Scientific, Inc., Yokohama, Japan). Thereafter, 45 individual clones were sequenced with an ABI PRISM ${ }^{\circledR} 310$ sequencer (Thermo Fisher Scientific, Inc.) using primers shown in Table I. Fig. 2 shows the promoter region of the FoxP3 gene. There are several transcription factor binding sites such as nuclear factor of activated $\mathrm{T}$ cells (NFAT) and activator protein 1 (AP-1), as well as promoter sequences such as CAAT, GC and TATA boxes. Ten CpG sites are located at positions -419, -256, -216, -139, $-127,-114,-78,-66,-59,-44$ and -16 . Following bisulfate treatment, unmethylated cytosine is converted to uracil, however, methylated cytosine is not converted. Thus, after sequencing, the extent of $\mathrm{C}$ in the clones indicates the original methylation status of each CpG site.

To monitor expression of FoxP3 in Org and subline CB1, $0,1,5$ or $10 \mu \mathrm{M}$ of demethylating agent 5-aza-deoxycytidine 
Table I. Primers used for real-time RT-PCR and cloning.

Sequences

\begin{tabular}{llc}
\hline & \multicolumn{2}{c}{ Sequences } \\
\cline { 2 - 3 } Gene & \multicolumn{1}{c}{ Forward } & Reverse \\
\hline For RT-PCR & & \\
FoxP3 & 5'-TCCCAGAGTTCCTCCACAAC-3' & 5'-TGTTCGTCCATCCTCCTTTC-3' \\
CTLA4 & 5'-TGACAGCCAGGTGACTGAAG-3' & 5'-ATGAGCTCCACCTTGCAGAT-3' \\
GITR & 5'-GAGGAGTGCTGTTCCGAGTG-3' & 5'-TCTGTCCAAGGTTTGCAGTG-3' \\
DNMT1 & 5'-GTGGGGGACTGTGTCTCTGT-3' & 5'-TGAAAGCTGCATGTCCTCAC-3' \\
DNMT2 & 5'-CCAAAGTCATTGCTGCGATA-3' & 5'-TCAGGAAATCCGAACTCTGG-3' \\
DNMT3a & 5'-ATCTCCAAGTCCCCATCCAT-3' & 5'-CAGCCATTTTCCACTGCTCT-3' \\
DNMT3b & 5'-CCCATTCGAGTCCTGTCATT-3' & 5'-GGTTCCAACAGCAATGGACT-3' \\
IKZF2 & 5'-AAAACAACATGGATGGCCCC-3' & 5'-GGGCTTTGTTTCCTCTTGGG-3' \\
SATB1 & 5'-TCAGTGGAAGCCTTGGGAAT-3' & 5'-TTGTCCTTCAGTTTGCCGTG-3' \\
AML1 & 5'-CCCTAGGGGATGTTCCAGAT-3' & 5'-TGAAGCTTTTCCCTCTTCCA-3' \\
For cloning & & \\
FoxP3 & 5'-GGATCCACCGTACAGCGTGGTTTTTC-3' & 5'-GAATTCTGTTCGTCCATCCTCCTTTC-3' \\
\hline
\end{tabular}

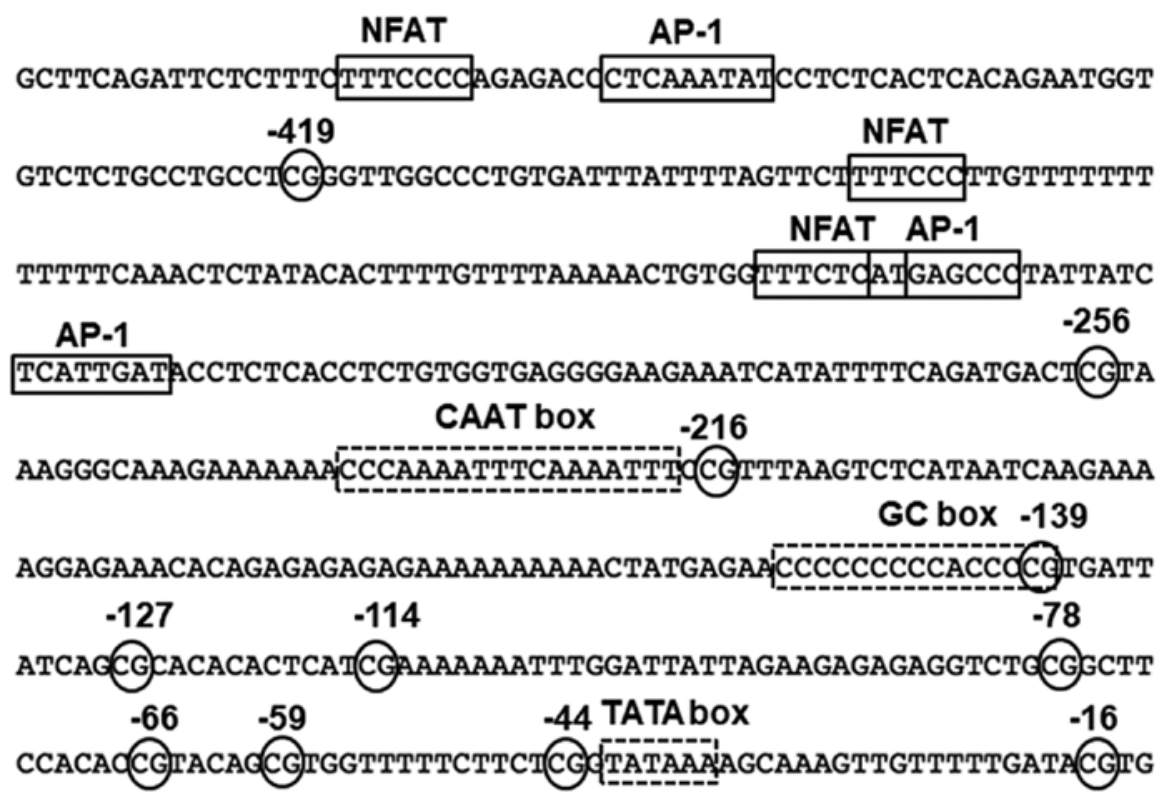

ACAGTTTCCCACAAGCCAGGCTGATCCTTTTCTGTCAGTCCACTTCACCAAGGT

Figure 2. Sequence of the promoter region of the FoxP3 gene. There are several transcription factor binding sites indicated by solid lines and transcription initiating sequences shown by dotted lines. Additionally, there are $11 \mathrm{CpG}$ sites (indicated by circles) that could be examined by the methylation assay.

(5-aza-dC) was added to cell cultures for 5 days and real-time RT-PCR assays were performed.

Statistical analysis. Statistical analysis for the expression levels assayed by real-time RT-PCR was performed using SPSS for Windows, version 21 (IBM Japan, SPSS Statistics, Tokyo, Japan) by Fisher's PLSD test after ANOVA analysis and the data of $\log _{10}$ values of relative expression.

\section{Results}

FoxP3 expression in MT-Org and sublines. As shown in Fig. 3A, relative mRNA expression was higher in Org compared with sublines CA1 to 3 and CB1 to 3 continuously exposed to asbestos. Protein expression was assayed by western blotting (Fig. 3B) using Org and sublines CA1 to 3, CB1 to 3, and $\mathrm{CR} 1$. The use of three different monoclonal antibodies revealed different expression patterns as indicated by $\mathrm{Ab} \mathrm{A}, \mathrm{B}$ and $\mathrm{C}$. It seemed that there was no significant difference in FoxP3 protein expression between Org and sublines as determined by our western blotting approach. Furthermore, flow cytometric analysis of intranuclear FoxP3 expression revealed similar levels between Org and sublines (CB1 to 3) as shown in Fig. 3C.

Expression of Treg-specific cell surface molecules. The cell surface molecules CTLA4 and GITR are known to be 
FoxP3 expression

A Relative mRNA levels

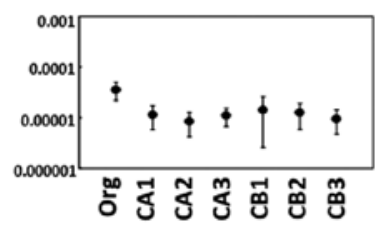

C



Intranuclear expression
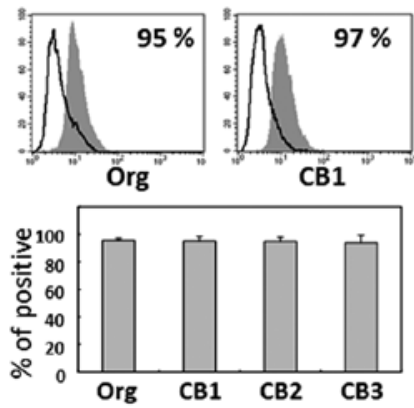

B
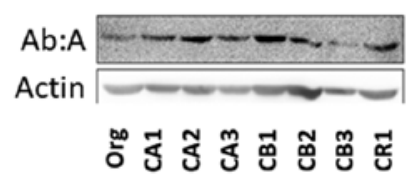

$\mathrm{Ab}: \mathrm{B}$

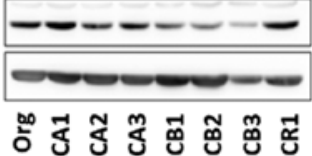

$\mathrm{Ab}: \mathrm{C}=-\ldots-\infty$
Actin

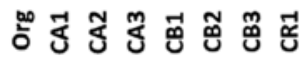
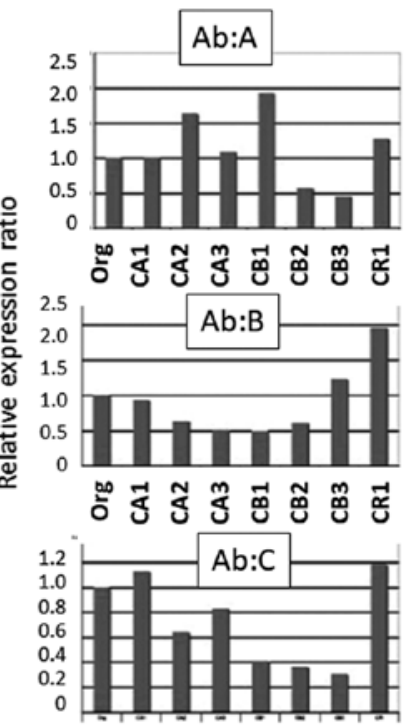



Figure 3. FoxP3 expression analyzed in Org and sublines. (A) Examination of relative mRNA expression assayed by real-time RT-PCR revealed significantly reduced expression in all sublines. (B) Although western blotting using three different monoclonal antibodies from different companies did not show any clear changes or shifts in expression, (C) flow cytometric assays for intranuclear FoxP3 showed almost identical full expression of FoxP3 amongst Org and sublines CB1 to 3 .

CTLA4
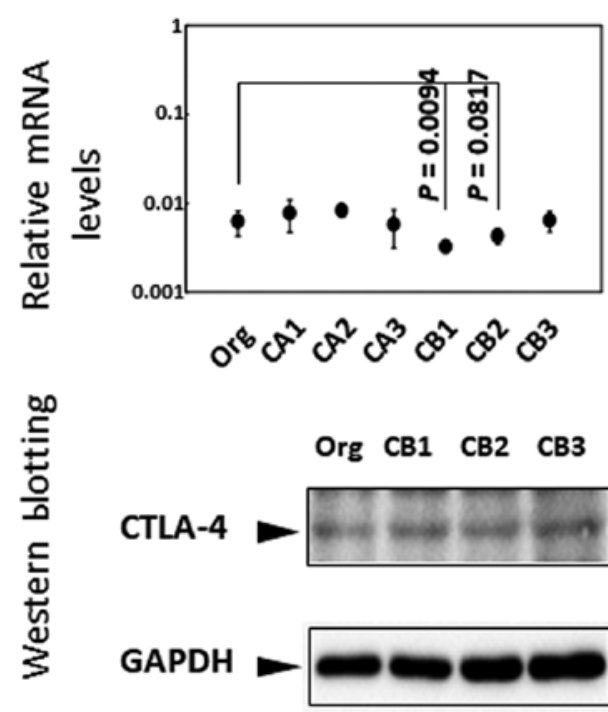

GITR

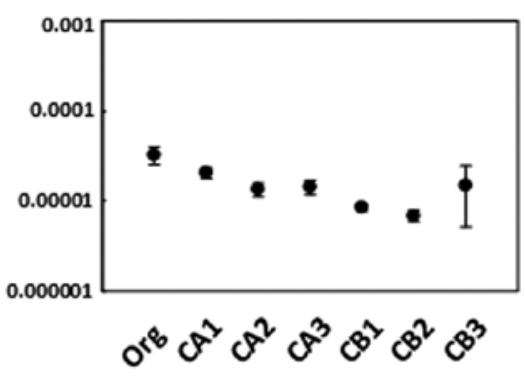

Org CB1 CB2 CB3

GITR

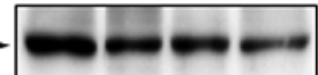

GAPDH

P-value obtained using Fisher's PLSD test.

Figure 4. Expression of Treg-specific molecules CTLA4 and GITR in Org and sublines. Messenger RNA expression in sublines CB1 and CB2 showed significantly decreased expression of CTLA-4, although other sublines did not show any significant changes. For the analysis of CTLA4 protein expression, western blotting and flow cytometric assays were performed and sublines CB1 to 3 tended to show an increase in CTLA4 expression as determined by flow cytometry, although the changes were not significant. Real-time RT-PCR and western blotting were performed for the investigation of GITR expression. As a result, no differences were found between Org and sublines. GITR was almost fully expressed in all lines examined, and may be due to the nature of the cell lines examined.

expressed in Treg cells $(34,35)$. Thus, the expression patterns of both molecules were examined in terms of mRNA levels, western blotting and flow cytometry. As shown in Fig. 4, the relative mRNA expression of CTLA4 was lower in CB1 and CB2 sublines, while other sublines examined showed no differences. Although western blot analysis also showed no significant changes, flow cytometric analyses showed higher expression in sublines CB1 to 3 compared with Org (there was 


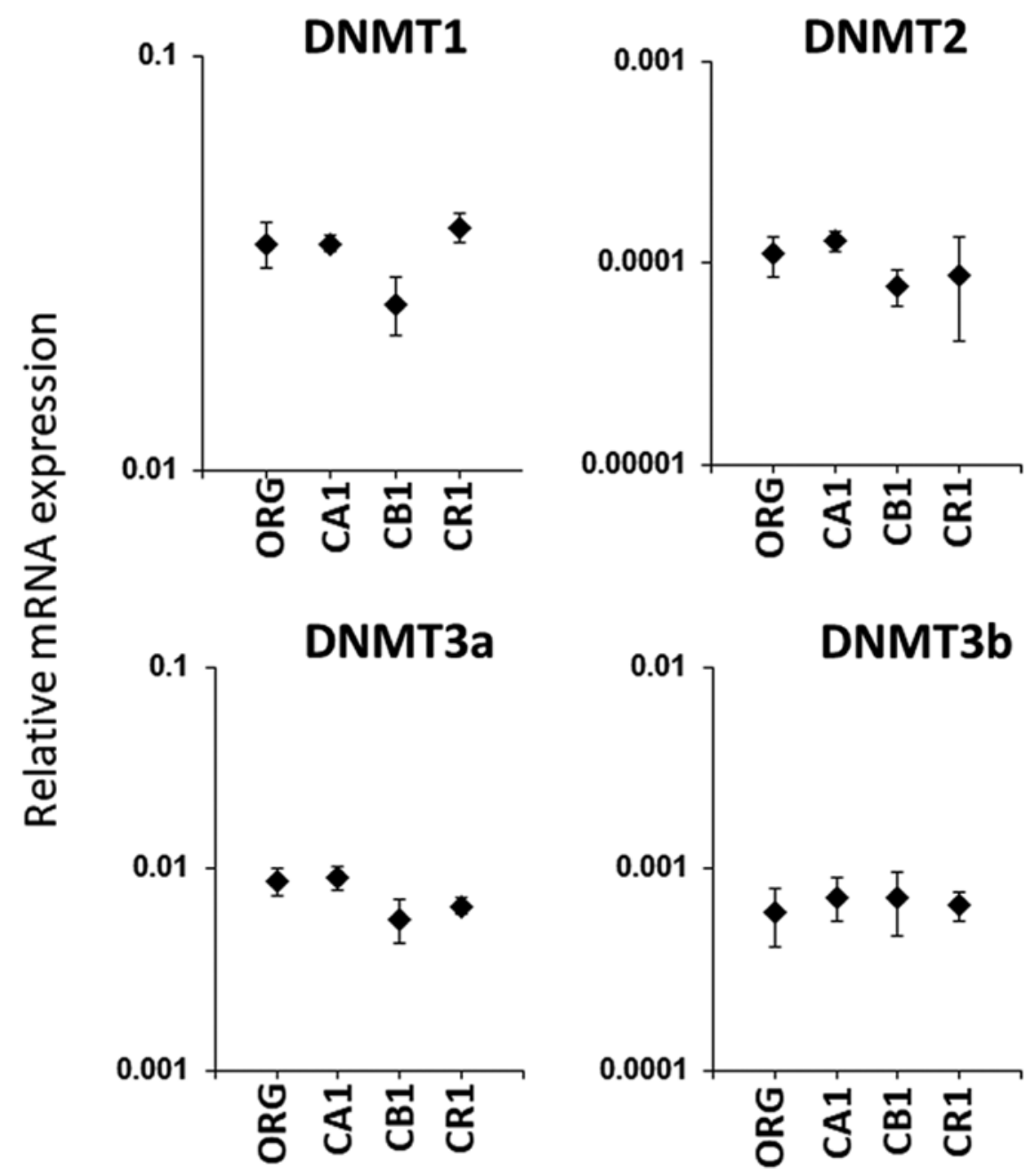

Figure 5. Messenger RNA expression of methyltransferases (DNMT1, 2, 3a and 3b) is examined using real-time RT-PCR in Org and three sublines (CA1, CB1 and CR1). Although it was found that expression levels varied, there was no statistical significance.

no statistical significance). Similar to CTLA4, there were no significant differences found in the expression of GITR between Org and sublines (CA1 to 3 and CB1 to 3) in terms of relative mRNA expression, protein expression assayed by western blotting or flow cytometry [(the results of flow cytometry were previously published (29)].

mRNA expression of methyltransferase. As previously published, sublines of MT-2 continuously exposed to asbestos revealed enhanced Treg function by cell-cell contact as well as an increase in typical soluble factors such as IL-10 and TGF- $\beta$, and expression of the master transcription factor of Treg FoxP3 was hypothesized to be enhanced. However, as shown in Fig. 3A, mRNA levels in the sublines were decreased. In an effort to delineate the mechanisms responsible for the apparent discrepancy between enhanced function and reduced mRNA levels, the methylation status of FoxP3 was compared between Org and sublines. Prior to examining $\mathrm{CpG}$ methylation in the promoter region of the FoxP3 gene, the relative mRNA expression of four different methyltransferases was assayed. As shown in Fig. 5, examination of methyltransferases DNMT1, DNMT2, DNMT3a and DNMT3b did not reveal any significant differences between Org and sublines CA1, CB1 and CR1.
Cp $G$ methylation status in FoxP3 gene promoter region in Org and sublines. As shown in Fig. 6, the methylation status of eleven $\mathrm{CpG}$ sites located in the promoter region of the FoxP3 gene was examined in MT-2 original cells (Org) as well as in sublines CA1, CB1 and CR1 continuously exposed to asbestos. Subline CB1 showed a relatively hypomethylated state in four 5' CpG sites located at positions $-419,-256,-216$ and $-139 \mathrm{CpG}$. However, these were CB1-specific, and were not found in sublines CA1 or CR1. The most hypomethylated $\mathrm{CpG}$ region found was located at position -216 in all cell lines examined, with values of $77 \%$ in Org, $25 \%$ in CA1, $11 \%$ in $\mathrm{CB} 1$ and $53 \%$ in CR1. Although the methylation status of this -216 site was the lowest in Org, the difference between Org and other sublines was the most marked. Hypomethylation of the $-216 \mathrm{CpG}$ region was greater in sublines continuously exposed to asbestos compared with Org. However, examination of relative mRNA expression (shown in Fig. 3A) revealed reduced expression of FoxP3 in sublines continuously exposed to asbestos compared with Org. Methylation of the FoxP3 promoter in sublines continuously exposed to asbestos was supposed to be greater compared with Org. Notwithstanding the level of FoxP3 expression in Org, most of the CpG sites in the promoter region of FoxP3 in Org were methylated. Thus, 

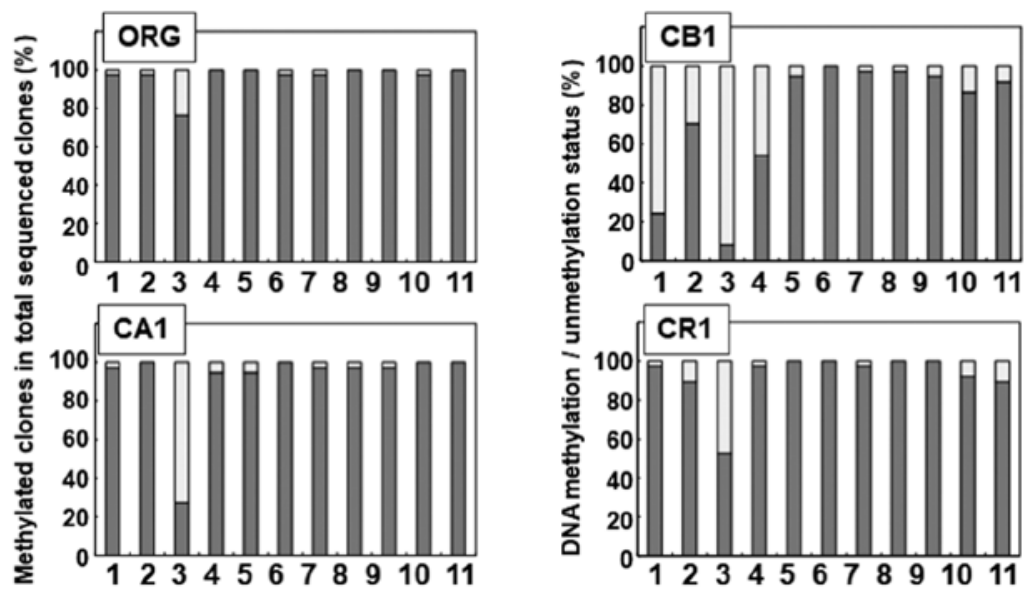

DNA methylation status (\%)

\begin{tabular}{cccccccccccc}
\hline Promoter & $\begin{array}{c}-419 \\
(1)\end{array}$ & $\begin{array}{c}-256 \\
(2)\end{array}$ & $\begin{array}{c}-216 \\
(3)\end{array}$ & $\begin{array}{c}-139 \\
(4)\end{array}$ & $\begin{array}{c}-127 \\
(5)\end{array}$ & $\begin{array}{c}-114 \\
(6)\end{array}$ & $\begin{array}{c}-78 \\
(7)\end{array}$ & $\begin{array}{c}-66 \\
(8)\end{array}$ & $\begin{array}{c}-59 \\
(9)\end{array}$ & $\begin{array}{c}-44 \\
(10)\end{array}$ & $\begin{array}{c}-16 \\
(11)\end{array}$ \\
\hline ORG & 98 & 98 & 77 & 100 & 100 & 98 & 98 & 100 & 100 & 98 & 100 \\
CA1 & 98 & 100 & 25 & 95 & 95 & 100 & 98 & 98 & 98 & 100 & 100 \\
CB1 & 27 & 72 & 11 & 56 & 95 & 100 & 98 & 98 & 95 & 87 & 93 \\
CR1 & 98 & 90 & 53 & 98 & 100 & 100 & 98 & 100 & 100 & 93 & 90 \\
\hline
\end{tabular}

Figure 6. Analysis of the methylation status of $\mathrm{CpG}$ sites found in the promoter region of the FoxP3 gene in Org and sublines CA1, CB1 and CR1 continuously exposed to asbestos. The upper panel shows a bar-graph from the lower panel table. \#(1) to (11) represent the eleven CpG sites located at positions - 419 to -16. In the upper bar graph, the y-axis shows the percentage of methylated clones in the total analyzed clones following bisulfate treatment and subsequent sequencing. The only $\mathrm{CpG}$ site in Org with a value $<80 \%$ was \#(3) located at position -216 . This site was also much more hypomethylated in sublines, with values of 25,11 and 53\% in CA1, CB1 and CR1, respectively. Although CA1 and CR1 showed hypomethylated CpG sites only in \#(3) at position -216, CB1 showed hypomethylated states in \#(1) at -419 with $27 \%$,\#(2) at -256 with $72 \%$, and \#(4) at -139 with $56 \%$, in addition to \#(3) as mentioned above. However, almost all of the CpG sites in the promoter region of FoxP3 in Org as well as sublines examined were methylated. The methylation status of \#(3) at -216 was lower in sublines compared with Org. However, this difference was not amenable to mRNA expression analysis shown in Fig. 3, as FoxP3 mRNA expression was lower in sublines compared with Org.
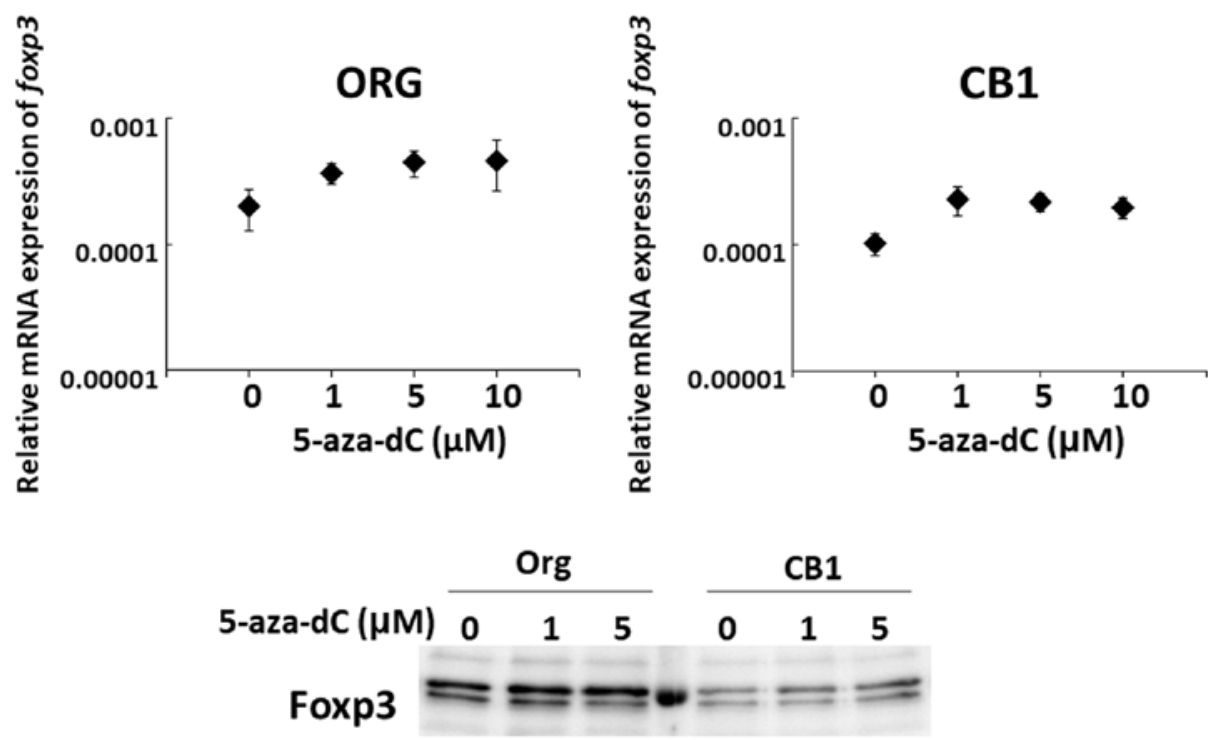

Histon $\mathrm{H} 4$

Figure 7. As shown in Fig. 6, the methylation status of $\mathrm{CpG}$ sites in the promoter region of the FoxP3 gene in Org and subline CB1 did not show marked differences and most of the sites were hypermethylated. In an effort to confirm that these methylation states were affected by demethylating agents, Org and subline $\mathrm{CB} 1$ cells were cultured in the absence or presence of 1,5 or $10 \mu \mathrm{M} 5$-aza-dC. Thereafter, FoxP3 mRNA expression was examined. Even though there was no statistical significance, it seemed that mRNA expression (upper panel) tended to be upregulated following 5-aza-dC treatment in Org and CB1 lines. This same pattern was observed for protein expression as examined by western blotting (lower panel), again with no statistical significance. 


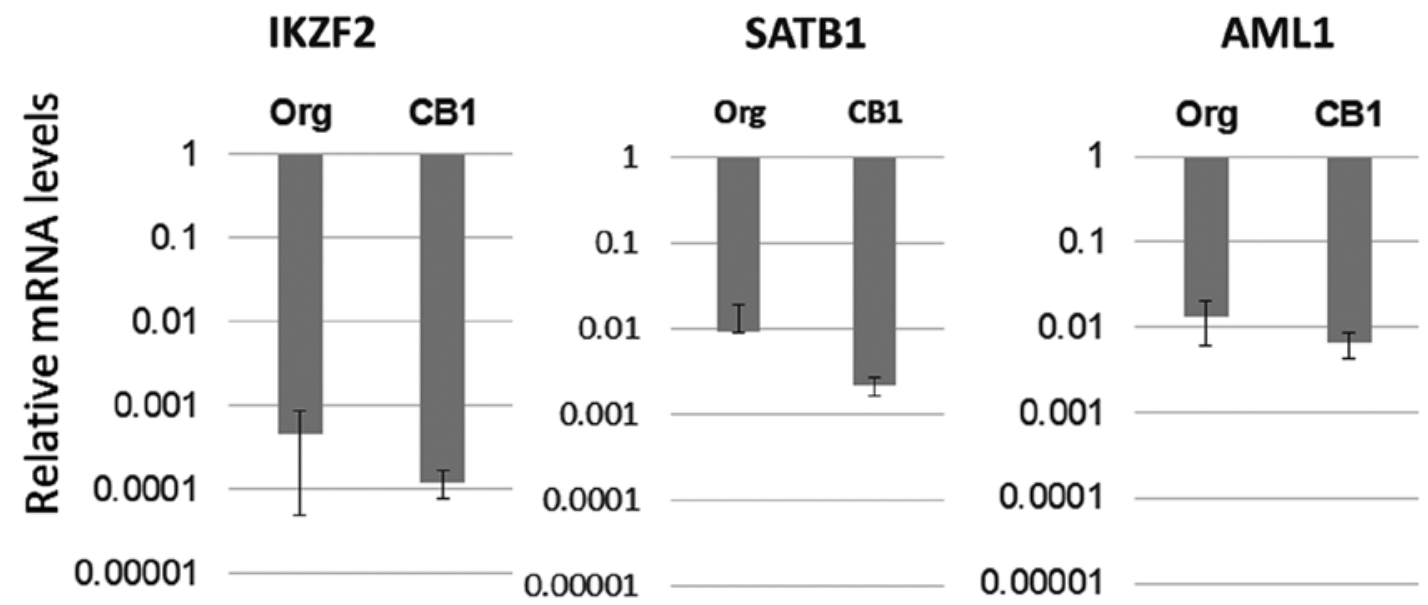

Figure 8. Messenger RNA expression of other transcription factors (IKZF2, SATB1 and AML1) considered important for FoxP3 was examined by real-time RT-PCR. There were no significant differences between Org and CB1 cells for all three genes examined.

reduced mRNA expression would not be caused by hypermethylation.

Effect of 5-aza-dC on changes in mRNA expression in Org and subline CB1. Although expression levels of FoxP3 in Org and sublines were insufficient for the RT-PCR and methylation assays, the effect of the demethylating agent 5-aza-deoxycytidine (5-aza-dC) was examined. As shown in Fig. 7, although there was no statistical significance, mRNA as well as protein expression seemed to increase following treatment with 5-aza-dC.

Expression of Treg-specific transcription factors other than FoxP3 in Org and subline CB1. Since Treg function in MT-2 sublines is enhanced following continuous exposure to asbestos, it was considered that expression of FoxP3, the key transcription factor in Treg cells, may be enhanced. However, FoxP3 mRNA levels were lower in sublines compared with Org. Furthermore, marked changes in protein levels were absent and the methylation of $\mathrm{CpG}$ sites in the promoter region of FoxP3 was much greater in Org than in sublines. As a result, mRNA expression of other important transcription factors in Treg cells such as IKZF2, SATB1 and AML1 was examined in Org and subline CB1. As shown in Fig. 8, there were no significant differences between Org and subline CB1 for each of the three transcription factors examined.

\section{Discussion}

Given the occurrence of malignant tumors such as lung cancer and MM in individuals exposed to asbestos, investigation of the carcinogenic activity of asbestos fibers themselves has been a major focus (1-11). Inhaled fibers remain in the pulmonary and pleural regions and their cancer-inducing properties continuously influence surrounding lung epithelial and pleural mesothelial cells. For example, DNA damage is caused by ROS and reactive nitrogen species (RNS) produced by iron contained in certain asbestos fibers, especially crocidolite and amosite $(8,9)$, as well as fiber-untreated frustrated alveolar macrophages. Direct damage to chromosomes due to entrance of asbestos fibers into cell bodies can also result in genomic damage. Furthermore, adsorption of various carcinogenic substances included in tobacco smoke and air pollution can also lead to malignant transformation (1-11).

Additionally, given the carcinogenic activity of asbestos fibers, we have been investigating the biological effects of asbestos fibers on human immune cells and have examined the resulting decrease in antitumor immunity (12-14). As previously reported, NK cell activity was reduced with decreased expression of activating receptor NKp46 $(15,16)$. The clonal expansion of CTL was also inhibited by culturing with asbestos fibers (17). Furthermore, intracellular perforin levels decreased in peripheral blood $\mathrm{CD}^{+}$cells derived from $\mathrm{MM}$ patients (40). Chemokine receptor CXCR3 levels were reduced in freshly isolated peripheral $\mathrm{CD}^{+}$cells continuously exposed to asbestos fibers during stimulation, as well as in $\mathrm{CD}^{+}$cells from PP and MM patients $(18,19)$. Additionally, CD4 ${ }^{+} \mathrm{T}$ cells from MM patients showed decreased intracellular IFN- $\gamma$ content following in vitro stimulation $(18,19)$. All of these findings indicated that individuals exposed to asbestos show a gradual decrease in antitumor immunity with subsequent onset of cancer due to a reduction in tumor surveillance activity, in addition to a rapid progression of the cancers (12-14).

When considering antitumor immunity, the function and volume of Treg cells are also important since Treg cells suppress tumors by attacking $\mathrm{T}$ cells which recognize tumor antigens (20-22). Thus, patients with enhanced function and volume of Treg cells show weakened antitumor immunity. We investigated Treg function and volume using a cell line model. In this model, we employed the MT-2 cell line with continuous exposure to relatively low doses of asbestos as this cell line was reported to possess Treg-like function $(23,24)$. As previously reported, MT-2 cells exposed to asbestos fibers showed enhanced Treg function via cell-cell contact as well as increased production of typical soluble factors for Treg function such as IL-10 and TGF- $\beta(28,29)$. Furthermore, cell cycle progression in MT-2 sublines was enhanced compared with Org as a result of reduced levels of CDK-Is and elevated levels of cyclins regulated by a marked decrease in FoxO1 transcription factor in an examined subline, as FoxO1 is known to regulate CDK-Is and cyclins in a positive and negative manner, respectively $(30,31)$. 
After obtaining these findings, it was thought that expression of FoxP3, the key transcription factor in Treg cells (20-22), may be enhanced in sublines continuously exposed to asbestos. However, as shown in Fig. 3, FoxP3 expression varied, as determined by examination of mRNA and protein levels. Relative mRNA levels were reduced, while marked or specific changes in protein levels were absent. Levels of other Treg-specific surface molecules such as CTLA4 and GITR also remained more or less unchanged, while a slight increase in CTLA4 was observed in a subline. These results may reflect the nature of the MT-2 cell line. During long-term cultivation, expression levels of molecules in cell lines may be altered compared with the respective normal cell counterpart, even though MT-2 is not a tumor-derived cell line but a virus-immortalized cell line.

Since there was a difference in FoxP3 expression between Org and sublines, the methylation status of the FoxP3 promoter region was assayed. Again, the results were unexpected since it was found that most of the $\mathrm{CpG}$ sites in the FoxP3 promoter region in Org were methylated. The lowest methylation was located at position -216, although $77 \%$ of clones were still methylated. Additionally, although mRNA expression was lower compared with Org, subline CB1 showed reduced methylation at positions $-419,-256,-216$ and -139 , while sublines CA1 and CR1 showed decreased methylation at the $-216 \mathrm{CpG}$ site. Furthermore, treatment with the demethylating agent 5-aza-dC caused a slight increase in FoxP3 mRNA expression as well as protein expression in Org and subline CB1. These results fail to clarify our understanding of the apparent discrepancy between functional enhancement of sublines as Treg-like cells and expression of FoxP3, the key transcription factor in Treg cells. RT-PCR was then employed in an effort to identify other transcription factors important in Treg cells, such as IKZF2, SAATB1 and AML1, whose expression may change following exposure to asbestos. However, no significant differences were found between Org and subline CB1 for any of the three genes examined. Additionally, we examined the effect of trichostatin A (TSA) (41), a typical histone deacetylase inhibitor (HDI or HDACI), on the Treg function of sublines, however, no changes were found (data not shown). Thus, epigenetic modifications are not important for Treg function enhancement in MT-2 sublines continuously exposed to asbestos.

Collectively, all examinations performed in this study indicated aberrant expression of FoxP3 in MT-2-derived sublines possessing enhanced Treg cell-like function and continuously exposed to asbestos fibers. Furthermore, other Treg-specific transcription factors did not show any marked changes with continuous exposure to asbestos. Thus, the precise nature of the cellular and molecular modifications responsible for the enhanced Treg function in MT-2 sublines continuously exposed to asbestos remains unknown. One possibility involves changes in cell surface molecules related to cell-cell contact to suppress effector $\mathrm{T}$ cell proliferation. From a protein expression assay previously reported, sublines examined showed increased phosphorylation of $\beta$-actin as well as cytoskeletal molecules including vimentin, myosin- 9 and tubulin- $\beta 2$, and showed asbestos fiber-binding activity when assayed under cell-free fiber-protein binding conditions (41). In one of our previous investigations, it was concluded that overproduction of IL-10 was regulated by Src-kinase since use of a Src inhibitor resulted in reduced IL-10 production in Org and subline $\mathrm{CB} 1$. Thus, one possible mechanism responsible for enhanced function in MT-2 sublines involves increased production of soluble factors such as IL-10 and TGF- $\beta(28,29)$. This may not be directly caused by the action of Treg-specific transcription factors. Another mechanism that may play a role involves cell-cell contact to suppress effector $\mathrm{T}$ cell proliferation. These interactions may be mediated by molecules located on the cell surface.

Additionally, there are discrepancies between gene and protein expression as well as function resembling Treg in MT-2 sublines continuously exposed to asbestos. Thus, there may be some modification regarding the ubiquitin-proteasome system in these sublines. Although given that in one of our previous experimental trials in which addition of the typical proteasome inhibitor bortezomib $(42,43)$ to subline cultures was found to have no effect on Treg function (data not shown), more detailed examinations may be required.

Thus, further analyses focusing on cell surface molecules in MT-2 sublines are required to delineate the precise manner by which continuous exposure to asbestos fibers causes enhanced Treg function. Thereafter, it could be confirmed whether certain cell surface molecules found to be altered in sublines are similarly altered in peripheral blood Treg cells from asbestos-exposed cell populations such as those from PP and MM patients, as well as Treg cells surrounding mesothelioma or asbestos-induced lung cancer cells. Thereafter, these could be targets in strategies to recover antitumor immunity in asbestos-exposed individuals and may lead to the prevention of asbestos-induced cancers.

\section{Acknowledgements}

The authors thank Ms. Naomi Miyahara, Tamayo Hatayama, Shoko Yamamoto and Miho Ikeda for their technical assistance.

\section{Funding}

Parts of this study were supported by The Special Coordination Funds for Promoting Science and Technology Grant H18-1-3-3-1 (Comprehensive Approach on Asbestos-Related Diseases), Grants-in-Aid for Scientific Research-KAKENHI-(nos. 20390178 and 22700933) and Kawasaki Medical School Project Grants (22-A58 and 29P001).

\section{Availability of data and materials}

All data generated or analyzed during this study are included in this published article.

\section{Authors' contributions}

MM mainly planed and performed methylation and expression (RT-PCRs and western blotting) analyses. HM performed western blot analysis. SY and SL performed and supported RT-PCR analysis. NKT, KY, YM and NS discussed all the performed findings depend on our hypothesis and before writing manuscript. MM and YN were major contributors for writing manuscript. TO comprehensively organized the 
projects regarding the immunological effects of asbestos. All authors read and approved the manuscript and agree to be accountable for all aspects of the research in ensuring that the accuracy or integrity of any part of the work are appropriately investigated and resolved.

\section{Ethics approval and consent to participate}

Not applicable.

\section{Patient consent for publication}

Not applicable.

\section{Competing interests}

The authors declare that they have no competing interests.

\section{References}

1. Attanoos R: Lung cancer associated with asbestos exposure. In Asbestos and Its Diseases. Craighead JE and Gibbs AR (eds). Oxford University Press, New York, pp172-189, 2008.

2. Hammar SP: Asbestos and mesothelioma. In: Sebstos; Risk Assessment, Epidemiology, and Health Effects. Dodson RF and Hammar SP (eds). 2nd edition. CRC Press, Boca Raton, FL, pp307-418, 2011.

3. Asbestos and Mesothelioma (Current Cancer Research) Testa JR (ed). Springer International Publishing, Gewerbestrasse, Switzerland, 2017.

4. International Agency for Research on Cancer: A review of human carcinogens: Arsenic, metals, fibres, and dusts (iarc monographs on the evaluation of the carcinogenic risks to humans). WHO Press, Lyon, France. 2012.

5. Shukla A, Gulumian M, Hei TK, Kamp D, Rahman Q and Mossman BT: Multiple roles of oxidants in the pathogenesis of asbestos-induced diseases. Free Radic Biol Med 34: 1117-1129, 2003.

6. Upadhyay D and Kamp DW: Asbestos-induced pulmonary toxicity: Role of DNA damage and apoptosis. Exp Biol Med 228 650-659, 2003.

7. Liu X and Chen Z: The pathophysiological role of mitochondrial oxidative stress in lung diseases. J Transl Med 15: 207, 2017.

8. Simeonova PP and Luster MI: Iron and reactive oxygen species in the asbestos-induced tumor necrosis factor-alpha response from alveolar macrophages. Am J Respir Cell Mol Biol 12: 676-683, 1995.

9. Boyles MS, Young L, Brown DM, MacCalman L, Cowie H, Moisala A, Smail F, Smith PJ, Proudfoot L, Windle AH, et al: Multi-walled carbon nanotube induced frustrated phagocytosis, cytotoxicity and pro-inflammatory conditions in macrophages are length dependent and greater than that of asbestos. Toxicol In Vitro 29: 1513-1528, 2015.

10. Toyokuni S: Mechanisms of asbestos-induced carcinogenesis. Nagoya J Med Sci 71: 1-10, 2009.

11. Chew SH and Toyokuni S: Malignant mesothelioma as an oxidative stress-induced cancer: An update. Free Radic Biol Med 86: 166-178, 2015

12. Otsuki T, Maeda M, Murakami S, Hayashi H, Miura Y, Kusaka M, Nakano T, Fukuoka K, Kishimoto T, Hyodoh F, et al: Immunological effects of silica and asbestos. Cell Mol Immunol 4: 261-268, 2007.

13. Kumagai-Takei N, Maeda M, Chen Y, Matsuzaki H, Lee S, Nishimura Y, Hiratsuka $\mathrm{J}$ and Otsuki T: Asbestos induces reduction of tumor immunity. Clin Dev Immunol 2011: 481439, 2011.

14. Otsuki T, Matsuzaki H, Lee S, Kumagai-Takei N, Yamamoto S, Hatayama T, Yoshitome K and Nishimura Y: Environmental factors and human health: Fibrous and particulate substance-induced immunological disorders and construction of a health-promoting living environment. Environ Health Prev Med 21: 71-81, 2016.
15. Nishimura Y, Miura Y, Maeda M, Kumagai N, Murakami S, Hayashi H, Fukuoka K, Nakano T and Otsuki T: Impairment in cytotoxicity and expression of NK cell-activating receptors on human NK cells following exposure to asbestos fibers. Int J Immunopathol Pharmacol 22: 579-590, 2009.

16. Nishimura Y, Maeda M, Kumagai N, Hayashi H, Miura Y and Otsuki T: Decrease in phosphorylation of ERK following decreased expression of NK cell-activating receptors in human NK cell line exposed to asbestos. Int J Immunopathol Pharmacol 22: 879-888, 2009.

17. Kumagai-Takei N, Nishimura Y, Maeda M, Hayashi H, Matsuzaki H, Lee S, Hiratsuka J and Otsuki T: Effect of asbestos exposure on differentiation of cytotoxic T lymphocytes in mixed lymphocyte reaction of human peripheral blood mononuclear cells. Am J Respir Cell Mol Biol 49: 28-36, 2013.

18. Maeda M, Nishimura Y, Hayashi H, Kumagai N, Chen Y, Murakami S, Miura Y, Hiratsuka J, Kishimoto T and Otsuki T: Reduction of CXC chemokine receptor 3 in an in vitro model of continuous exposure to asbestos in a human T-cell line, MT-2. Am J Respir Cell Mol Biol 45: 470-479, 2011.

19. Maeda M, Nishimura Y, Hayashi H, Kumagai N, Chen Y, Murakami S, Miura Y, Hiratsuka J, Kishimoto T and Otsuki T: Decreased CXCR3 expression in $\mathrm{CD}^{+} \mathrm{T}$ cells exposed to asbestos or derived from asbestos-exposed patients. Am J Respir Cell Mol Biol 45: 795-803, 2011.

20. Chen $X$, Du Y, Lin X, Qian Y, Zhou $T$ and Huang Z: $\mathrm{CD} 4+\mathrm{CD} 25+$ regulatory $\mathrm{T}$ cells in tumor immunity. Int Immunopharmacol 34: 244-249, 2016.

21. Szylberg $€$, Karbownik D and Marszałek A: The role of foxP3 in human cancers. Anticancer Res 36: 3789-3794, 2016.

22. Tanaka A and Sakaguchi S: Regulatory T cells in cancer immunotherapy. Cell Res 27: 109-118, 2017.

23. Hamano R, Wu X, Wang Y, Oppenheim JJ and Chen X: Characterization of MT-2 cells as a human regulatory T cell-like cell line. Cell Mol Immunol 12: 780-782, 2015.

24. Chen S1, Ishii N, Ine S, Ikeda S, Fujimura T, Ndhlovu LC, Soroosh P, Tada K, Harigae H, Kameoka J, et al: Regulatory $\mathrm{T}$ cell-like activity of Foxp $3^{+}$adult $\mathrm{T}$ cell leukemia cells. Int Immunol 18: 269-277, 2006.

25. Hyodoh F, Takata-Tomokuni A, Miura Y, Sakaguchi H, Hatayama T, Hatada S, Katsuyama H, Matsuo Y and Otsuki T: Inhibitory effects of anti-oxidants on apoptosis of a human polyclonal T-cell line, MT-2, induced by an asbestos, chrysotile-A. Scand J Immunol 61: 442-448, 2005.

26. Miura Y, Nishimura Y, Katsuyama H, Maeda M, Hayashi H, Dong M, Hyodoh F, Tomita M, Matsuo Y, Uesaka A, et al: Involvement of IL-10 and $\mathrm{Bcl}-2$ in resistance against an asbestos-induced apoptosis of T cells. Apoptosis 11: 1825-1835, 2006.

27. Maeda M, Yamamoto S, Chen Y, Kumagai-Takei N, Hayashi H, Matsuzaki H, Lee S, Hatayama T, Miyahara N, Katoh M, et al: Resistance to asbestos-induced apoptosis with continuous exposure to crocidolite on a human T cell. Sci Total Environ 429: 174-182, 2012.

28. Maeda M, Chen Y, Hayashi H, Kumagai-Takei N, Matsuzaki H, Lee S, Nishimura Y and Otsuki T: Chronic exposure to asbestos enhances TGF- $\beta 1$ production in the human adult $\mathrm{T}$ cell leukemia virus-immortalized T cell line MT-2. Int J Oncol 45: 2522-2532, 2014.

29. Ying C, Maeda M, Nishimura Y, Kumagai-Takei N, Hayashi H, Matsuzaki H, Lee S, Yoshitome K, Yamamoto S, Hatayama T and Otsuki T: Enhancement of regulatory T cell-like suppressive function in MT-2 by long-term and low-dose exposure to asbestos. Toxicology 338: 86-94, 2015

30. Matsuzaki H, Lee S, Maeda M, Kumagai-Takei N, Nishimura Y and Otsuki T: FoxO1 regulates apoptosis induced by asbestos in the MT-2 human T-cell line. J Immunotoxicol 13: 620-627, 2016.

31. Lee S, Matsuzaki H, Maeda M, Yamamoto S, Kumagai-Takei N, Hatayama T, Ikeda M, Yoshitome K, Nishimura Y and Otsuki T: Accelerated cell cycle progression of human regulatory T cell-like cell line caused by continuous exposure to asbestos fibers. Int J Oncol 50: 66-74, 2017.

32. Maeda M, Yamamoto S, Hatayama T, Matsuzaki H, Lee $S$, Kumagai-Takei N, Yoshitome K, Nishimura Y, Kimura Y and Otsuki T: T cell alteration caused by exposure to asbestos. In: Biological Effects of Fibrous and Particulate Substances. Otsuki T, Holian A and Yoshioka Y (eds). Springer Japan, Tokyo, pp195-210, 2015. 
33. Kohyama N, Shinohara Y and Suzuki Y: Mineral phases and some reexamined characteristics of the international union against cancer standard asbestos samples. Am J Ind Med 30: 515-528, 1996.

34. Boden E, Tang Q, Bour-Jordan H and Bluestone JA: The role of CD28 and CTLA4 in the function and homeostasis of CD4+CD25+ regulatory T cells. Novartis Found Symp 252: 55-63, 2003.

35. Ko K, Yamazaki S, Nakamura K, Nishioka T, Hirota K, Yamaguchi T, Shimizu J, Nomura T, Chiba T and Sakaguchi S: Treatment of advanced tumors with agonistic anti-GITR $\mathrm{mAb}$ and its effects on tumor-infiltrating Foxp $3^{+} \mathrm{CD} 25^{+} \mathrm{CD} 4^{+}$regulatory T cells. J Exp Med 202: 885-891, 2005.

36. Thornton AM, Korty PE, Tran DQ, Wohlfert EA, Murray PE, Belkaid Y and Shevach EM: Expression of Helios, an Ikaros transcription factor family member, differentiates thymic-derived from peripherally induced $\mathrm{Foxp}^{+} \mathrm{T}$ regulatory cells. J Immunol 184: 3433-3441, 2010.

37. Grzanka J, Leveson-Gower D, Golab K, Wang XJ, Marek-Trzonkowska N, Krzystyniak A, Wardowska A, Mills JM, Trzonkowski P and Witkowski P: FoxP3, Helios, and SATB1: Roles and relationships in regulatory $\mathrm{T}$ cells. Int Immunopharmacol 16: 343-347, 2013.

38. Ono M, Yaguchi H, Ohkura N, Kitabayashi I, Nagamura Y, Nomura T, Miyachi Y, Tsukada T and Sakaguchi S: Foxp3 controls regulatory T-cell function by interacting with AML1/Runx1. Nature 446: 685-689, 2007.
39. Maeda M, Chen Y, Lee S, Kumagai-Takei N, Yoshitome K, Matsuzaki H, Yamamoto S, Hatayama T, Ikeda M, Nishimura Y and Otsuki T: Induction of IL-17 production from human peripheral blood $\mathrm{CD}^{+}$cells by asbestos exposure. Int J Oncol 50: 2024-2032, 2017

40. Kumagai-Takei N, Nishimura Y, Maeda M, Hayashi H, Matsuzaki H, Lee S, Kishimoto T, Fukuoka K, Nakano T and Otsuki T: Functional properties of $\mathrm{CD} 8^{+}$lymphocytes in patients with pleural plaque and malignant mesothelioma. J Immunol Res 2014: 670140, 2014.

41. Maeda M, Chen Y, Kumagai-Takei N, Hayashi H, Matsuzaki H, Lee S, Hiratsuka J, Nishimura Y, Kimura Y and Otsuki T: Alteration of cytoskeletal molecules in a human $\mathrm{T}$ cell line caused by continuous exposure to chrysotile asbestos. Immunobiology 218: 1184-1191, 2013.

42. Wang L, Tao R and Hancock WW: Using histone deacetylase inhibitors to enhance Foxp $3^{+}$regulatory T-cell function and induce allograft tolerance. Immunol Cell Biol 87: 195-202, 2009.

43. Bennett MK and Kirk CJ: Development of proteasome inhibitors in oncology and autoimmune diseases. Curr Opin Drug Discov Devel 11: 616-625, 2008. 